\title{
EVALUATION OF IUPAC LIMIT OF DETECTION AND ISO MINIMUM DETECTABLE VALUE - ELECTROCHEMICAL DETERMINATION OF LEAD
}

\author{
JÁN MOCÁK ${ }^{1}$, IVAN JANIGA ${ }^{2}$, ESTERA RÁBAROVÁ ${ }^{1}$ \\ ${ }^{I}$ Department of Chemistry, University of SS. Cyril and Methodius, \\ Nám. J. Herdu 2, Trnava, SK-917 01, Slovak Republic (jan.mocak@ucm.sk) \\ ${ }^{2}$ Department of Theoretical Bases of Mechanical Engineering, \\ Faculty of Mechanical Engineering, Slovak University of Technology, \\ Nám. slobody 17, SK-81231 Bratislava, Slovak Republic
}

\begin{abstract}
The way of calculating the limit of detection recommended by IUPAC is compared to the minimum detectable value used by ISO as one of the most important performance characteristics of a measurement process. In this work, theoretical analysis of both characteristics is given together with directions for their practical use. Calculations are exemplified using electrochemical trace analysis of lead in surface water.
\end{abstract}

Key words: Limit of detection, critical value, minimum detectable value, limit of quantification, determination of lead, voltammetric water analysis.

\section{Introduction}

Capability of detection is an important performance characteristic of a measurement process. In chemistry, a representative characteristic of any analytical method is the smallest concentration or the mass of the analyte (the analyzed sample component) that can be detected with a specified degree of certainty. The related quantity is the limit of detection, LOD, defined by International Union for Pure and Applied Chemistry (IUPAC, 1978) first of all as

$$
y_{D}=\mu_{b}+k_{D} \sigma_{b}
$$

where $y_{D}$ denotes the LOD in the signal domain, $\mu_{b}$ is the expected mean blank value (the analyte-free sample), $\sigma_{b}$ is the standard deviation of the blank and $k_{D}$ is a proportionality factor. The statistical quantities $\mu_{b}$ and $\sigma_{b}$ are related to a very large set of observations and are unknown therefore they were in chemical practice commonly approximated by the sample quantities - the arithmetic mean $\bar{y}_{b}$ of $n_{b}$ blank measurements, and the blank standard deviation $s_{b}$.

Together with the LOD further limits have also been invented and used in chemistry. Among them, the limit of quantification (or determination), LOQ, refers to the smallest analyte concentration or mass, which can be quantitatively analysed with a reasonable reliability by a given procedure. Its oldest, traditional definition (ACS 
COMMITTEE, 1980) is similar to that given for LOD in the signal domain, but the numerical factor $k_{Q}$ is used:

$$
y_{Q}=\mu_{b}+k_{Q} \sigma_{b}
$$

In practice however, a more important task is to know the concentration equivalents of the signal LOD and LOQ values. For this purpose the slope $q_{1}$ of the linear calibration line, $y=q_{0}+q_{1} x$, expressing the dependence of the signal $y$ on concentration $x$, is commonly used:

$$
\begin{aligned}
& \operatorname{LOD}=\left(y_{D}-\bar{y}_{b}\right) / q_{1}=k_{D} s_{b} / q_{1} \\
& \operatorname{LOQ}=\left(y_{Q}-\bar{y}_{b}\right) / q_{1}=k_{Q} s_{b} / q_{1}
\end{aligned}
$$

It is noteworthy that the calibration model should be written in statistics using the variables denoted by capital letters, i.e. $Y=q_{0}+q_{1} X$, but it is frequently ignored in chemical literature for the sake of simplicity.

The proportionality factors $k_{D}=3$ and $k_{Q}=10$ have been traditionally accepted in this approach therefore this way of the LOD and LOQ calculation will be designed in this paper as "traditional approach". It is imperfect with regard to contemporary statistical theory mainly due to the following drawbacks (MOCAK et al., 1997): (1) the normal distribution is used for typically small sets of observations, supposing equality $\mu_{b}=\bar{y}_{b}$ and $\sigma_{b}=s_{b}$, (2) an exact intercept value is considered in calibration, supposing $q_{0}=\bar{y}_{b}$, unaffected by random errors. Despite these mistakes, mainly due to its simplicity and a long term usage, the traditional approach has been still used even though newer, more correct ways have been described in the literature, especially in the documents recommended by IUPAC (CURRIE, 1994a, b; 1995; MOCAK et al., 1997).

Situation in this field is rather complicated also due to some terminological inconsistency introduced in the past. Some authors (demanding equality of the $\alpha$ and $\beta$ errors) used the proportionality factor 6 for the limit of detection and the factor of 3 assigned to the limit of decision; others suggested to keep the way of the LOD calculation as explained above (with $k_{D}=3$ ) and utilized the proportionality factor 6 for the limit of identification, LOI (alternatively named also as the limit of guarantee of purity). A more detailed discussion on this topic can be found e.g. in the IUPAC document (MOCAK et al., 1997).

The aim of this work is to explain in a statistically correct way the calculation of the LOD and further limits characterizing the measurement method in chemistry in the region of low concentrations (trace analysis) and, in addition, to compare these limits to analogical characteristics defined by International Standards Organization (ISO, 2002), which is nowadays universally accepted as a leading metrological institution in many branches of science and technology. Moreover, it will be recommended in the conclusion part of this work what performance characteristics should be implemented when evaluating measurements in chemical trace analysis and a guideline will be proposed regarding the use of appropriate terminology. 


\section{Theory}

\subsection{Limit of detection obtained by upper limit approach}

The upper limit approach, ULA, is fundamental newer way of the LOD and LOQ calculations. It is based on the following details (MOCAK et al., 1997; MOCAK and BOBROWSKI, 2000): (a) It makes use of the one-sided upper confidence limit for a future individual observation (GREEN, 1978; MASSART et al., 1988), which defines the one-sided prediction band in calibration for predicting the maximum possible signal value, induced by random errors in measurement. The signal value corresponding to the zero analyte concentration (the blank) is here used at the $100(1-\alpha) \%$ probability level. (b) As the most suitable, the significance level $(1-\alpha)=$ 0.99 was recommended, which guarantees the best agreement of this calculation procedure with the previous traditional approach. (c) The signal variance is assumed constant (homoscedastic) therefore the sample standard deviation is considered constant at zero as well as non-zero concentrations (but not too far from zero). Consequently, the residual standard deviation $s_{y x}$, expressing the regression error, is used instead of the blank standard deviation $s_{b}$. (d) The values of the multiplication factors $k_{D}$ and $k_{Q}$ are not fixed but depend on the number of experiments performed, which is reflected by the critical value of $t$ - distribution utilized in calculation. (e) The concentration LOD and LOQ values are estimated from the corresponding signals rigorously by regression using the inverse calibration model, $X=F^{-1}(Y)$. It means that the LOD and LOQ calculations are calibration model dependent.

The concentration LOD and LOQ values for the general straight line calibration model, $Y=q_{0}+q_{1} X$, are given as:

$$
\begin{aligned}
& \mathrm{LOD}=\left[\mathrm{t}(n-2,1-\alpha) s_{y x} / q_{1}\right]\left[1+1 / n+\bar{x}^{2} / \sum_{i=1}^{n}\left(x_{i}-\bar{x}\right)^{2}\right]^{1 / 2} \\
& \mathrm{LOQ}=3\left[\mathrm{t}(n-2,1-\alpha) s_{y x} / q_{1}\right]\left[1+1 / n+\bar{x}^{2} / \sum_{i=1}^{n}\left(x_{i}-\bar{x}\right)^{2}\right]^{1 / 2}
\end{aligned}
$$

where $n$ concerns the number of calibration points involved in the regression procedure, $t(n-2,1-\alpha)$ is the critical $t$ - value for the number of degrees of freedom (equal to $n-2$ ) and the significance level $0.99, x_{\mathrm{i}}$ is the $i$-th point of the concentration coordinate, $\bar{x}$ is the mean concentration of all points used in calibration. Residual standard deviation $s_{y x}$ represents the error in regression and is given by the squared deviation of $\hat{y}_{i}$, calculated in regression, from $y_{i}$, measured in calibration experiment:

$$
s_{y x}=\sqrt{\frac{1}{n-2} \sum_{i=1}^{n}\left(y_{i}-\hat{y}_{i}\right)^{2}}=\sqrt{\frac{1}{n-2} \sum_{i=1}^{n}\left(y_{i}-q_{0}-q_{1} x_{i}\right)^{2}}
$$

Using this approach, the multiplication factor $k_{D}$ is defined as:

$$
k_{D}(n, \alpha)=t(n-2,1-\alpha)\left[1+1 / n+\bar{x}^{2} / \sum_{i=1}^{n}\left(x_{\mathrm{i}}-\bar{x}\right)^{2}\right]^{1 / 2}
$$


With regard to equations (5) and (6) there exists a simple relation between two multiplication factors: $k_{\mathrm{Q}}(n, \alpha)=3 k_{\mathrm{D}}(n, \alpha)$.

If the calibration experiment is performed in the equidistant manner, it is possible to utilize the corresponding entries of Table 1 for a quick calculation of the LOD and LOQ. Moreover, the LOD and LOQ values can be then calculated in a way similar to the traditional approach using simple multiplication formulae:

$$
\mathrm{LOD}=k_{D}(n, \alpha) s_{y x} / q_{1} ; \quad \mathrm{LOQ}=k_{Q}(n, \alpha) s_{y x} / q_{1}=3 \mathrm{LOD}
$$

The $C(n)$ and $B(n)$ terms introduced in Table 1 depend exclusively on the concentration values and are independent of the signal values therefore they can be pre-calculated. The only condition to assure the validity of this simplified calculation procedure is to keep strictly the equidistant allocation of all calibration points, including the blank value $(x=0)$.

\subsection{Critical value and minimum detectable value}

A performance characteristic of any measurement process, characterizing the capability of detection, has been defined by (ISO, $2000 \mathrm{a}, \mathrm{b}$; 2007) as the minimum detectable value, MDV. Another characteristic defined by ISO is the critical value, $\mathrm{CV}$. According to ISO 11843-2, the concentration of analyte in the laboratory sample is named the state variable, $Z$, since it represents the state of the material being analyzed. An analyte-free material is considered to be in the basic state. The difference between the state variable, $Z$, and its value in the basic state is called the net state variable, denoted by $X$. The state variable or the net state variable cannot be observed directly, but they are related to an observable response variable, $Y$, via calibration function $F$, defined by the mathematical relationship $Y=F(Z)$ or $Y=F(X)$, representing the mathematical model of the measurement. In chemical calibration measurements, the response variable $Y$ is usually an instrument signal. The investigated analyte concentration is finally calculated by means of the evaluation function $F^{-1}$, which represents the inverse of the calibration function, $X=F^{-1}(Y)$.

The ISO critical value, $x_{C}$, and the minimum detectable value, $x_{D}$, of the net state variable are defined (JANIGA et al., 2004, 2006) by the equations

$$
\begin{gathered}
\mathrm{CV}=x_{C}=\frac{y_{C}-\hat{q}_{0}}{\hat{q}_{1}}=t(v, 1-\alpha) \frac{\hat{\sigma}}{\hat{q}_{1}} \sqrt{1+\frac{1}{I J}+\frac{\bar{x}^{2}}{s_{x x}}} \\
\mathrm{MDV}=x_{D}=\delta(v, \alpha, \beta) \frac{\hat{\sigma}}{\hat{q}_{1}} \sqrt{1+\frac{1}{I J}+\frac{\bar{x}^{2}}{s_{x x}}}
\end{gathered}
$$

where $t(v, 1-\alpha)$ denotes a $(1-\alpha) \%$ quantile of the $t$ - distribution with $v=(I J-2)$ degrees of freedom, $I$ - number of states (calibration standards), $J$ - number of parallel measurements, $\delta$-non-centrality parameter of the non-central $t$-distribution, $\hat{\sigma}-$ estimated residual standard deviation of the calibration line (identical to $s_{y x}$ in part 2.1 for $n=I J)$. 
Table 1 . The $k_{D}$ factors and auxiliary quantities used in the LOD and LOQ calculation for $\alpha=0.01$ (onesided) and the number of experiments $n$ assuming equidistant calibration ${ }^{*}$

\begin{tabular}{|c|c|c|c|c|c|c|}
\hline$n$ & $\boldsymbol{v}$ & $(1+1 / n)^{1 / 2}$ & $C(n)$ & $B(n)$ & $t(v, 0.99)$ & $k_{D}(v, 0.99)$ \\
\hline 3 & 1 & 1.15470 & 0.50000 & 1.35401 & 31.821 & 43.086 \\
\hline 4 & 2 & 1.11803 & 0.45000 & 1.30384 & 6.965 & 9.081 \\
\hline 5 & 3 & 1.09545 & 0.40000 & 1.26491 & 4.541 & 5.744 \\
\hline 6 & 4 & 1.08012 & 0.35714 & 1.23443 & 3.747 & 4.625 \\
\hline 7 & 5 & 1.06905 & 0.32143 & 1.21008 & 3.365 & 4.072 \\
\hline 8 & 6 & 1.06066 & 0.29167 & 1.19024 & 3.143 & 3.741 \\
\hline 9 & 7 & 1.05409 & 0.26667 & 1.17379 & 2.998 & 3.519 \\
\hline 10 & 8 & 1.04881 & 0.24546 & 1.15994 & 2.897 & 3.360 \\
\hline 11 & 9 & 1.04447 & 0.22727 & 1.14812 & 2.821 & 3.239 \\
\hline 12 & 10 & 1.04083 & 0.21154 & 1.13792 & 2.764 & 3.145 \\
\hline 13 & 11 & 1.03775 & 0.19780 & 1.12904 & 2.718 & 3.069 \\
\hline 14 & 12 & 1.03510 & 0.18571 & 1.12122 & 2.681 & 3.006 \\
\hline 15 & 13 & 1.03280 & 0.17500 & 1.11430 & 2.650 & 2.953 \\
\hline 16 & 14 & 1.03078 & 0.16544 & 1.10813 & 2.624 & 2.908 \\
\hline 17 & 15 & 1.02899 & 0.15686 & 1.10258 & 2.602 & 2.869 \\
\hline 18 & 16 & 1.02740 & 0.14912 & 1.09758 & 2.583 & 2.836 \\
\hline 19 & 17 & 1.02598 & 0.14211 & 1.09304 & 2.567 & 2.806 \\
\hline 20 & 18 & 1.02470 & 0.13571 & 1.08891 & 2.552 & 2.779 \\
\hline 21 & 19 & 1.02353 & 0.12987 & 1.08512 & 2.539 & 2.756 \\
\hline 22 & 20 & 1.02247 & 0.12451 & 1.08165 & 2.528 & 2.734 \\
\hline 23 & 21 & 1.02151 & 0.11957 & 1.07844 & 2.518 & 2.715 \\
\hline 24 & 22 & 1.02062 & 0.11500 & 1.07548 & 2.508 & 2.698 \\
\hline 25 & 23 & 1.01980 & 0.11077 & 1.07274 & 2.500 & 2.682 \\
\hline 26 & 24 & 1.01905 & 0.10684 & 1.07019 & 2.492 & 2.667 \\
\hline 27 & 25 & 1.01835 & 0.10317 & 1.06781 & 2.485 & 2.654 \\
\hline 28 & 26 & 1.01770 & 0.09975 & 1.06558 & 2.479 & 2.641 \\
\hline 29 & 27 & 1.01709 & 0.09655 & 1.06350 & 2.473 & 2.630 \\
\hline 30 & 28 & 1.01653 & 0.09355 & 1.06155 & 2.467 & 2.619 \\
\hline 32 & 30 & 1.01550 & 0.08807 & 1.05800 & 2.457 & 2.600 \\
\hline 34 & 32 & 1.01460 & 0.08319 & 1.05480 & 2.449 & 2.583 \\
\hline 36 & 34 & 1.01379 & 0.07883 & 1.05200 & 2.441 & 2.568 \\
\hline 38 & 36 & 1.01307 & 0.07490 & 1.04940 & 2.435 & 2.555 \\
\hline 40 & 38 & 1.01242 & 0.07134 & 1.04710 & 2.429 & 2.543 \\
\hline$\infty$ & $\infty$ & 1.00000 & 0.00000 & 1.00000 & 2.326 & 2.326 \\
\hline
\end{tabular}

*) $t(v, 0.99)$ denotes the critical $t$-value; $\quad v=n-2 ; \quad B(n)=[1+1 / n+C(n)]^{1 / 2}$;

$$
C(n)=\bar{x}^{2} / \sum_{i=1}^{n}\left(x_{i}-\bar{x}\right)^{2}=1 / \sum_{i=1}^{n}\left(x_{i} / \bar{x}-1\right)^{2}
$$

Symbol $s_{x x}$ conventionally represents the sum

$$
s_{x x}=J \sum_{i=1}^{I}\left(x_{i}-\bar{x}\right)^{2}
$$


A considerable similarity of eqs. (5) and (10) is evident. Moreover, it should be noted that for the sake of simplicity (appreciated by a practical chemist) some symbols in the LOD calculation recommended by IUPAC (MOCAK et al., 1997) were simplified using e.g. $q_{0}, q_{1}$ and $s_{y x}$ instead of $\hat{q}_{0}, \hat{q}_{1}$ and $\hat{\sigma}$ (or $\hat{\sigma}_{y x}$ ); another change valid for this work is using $x$ for concentration instead of $c$.

\section{Material and methods}

All chemicals used were of Analytical Reagent Grade; distilled and de-ionised water was used in all measurements. Lead(II) ions in surface water were determined by differential pulse anodic stripping voltammetry (DPASV) using an AUTOLAB/PGSTAT 20 Electrochemical Instrument, The Netherlands. A standard three-electrode voltammetric cell with the static mercury drop electrode SMDE-1 (Laboratorní Př́istroje, Prague), a reference $3 \mathrm{~mol} / \mathrm{L}$ silver-silver chloride electrode, and a platinum wire auxiliary electrode were used in all electrochemical measurements. The following parameters were set for the $\mathrm{Pb}(\mathrm{II})$ determination: deposition potential $-1 \mathrm{~V}$, equilibration time $10 \mathrm{~s}$, modulation time $0.04 \mathrm{~s}$, interval time $0.1 \mathrm{~s}$, initial potential $-0.8 \mathrm{~V}$, end potential $-0.2 \mathrm{~V}$, step potential $-0.002 \mathrm{~V}$, modulation amplitude $-0.05 \mathrm{~V}$, and temperature $25 \pm 0.5{ }^{\circ} \mathrm{C}$. Experimental data sampled by the electrochemical instrument were written to the computer hard disk and finally processed by Microsoft EXCEL and ORIGIN (Microcal Software, Inc., Northampton, MA) software.

\section{Results and discussion}

\subsection{Comparison of IUPAC and ISO measurement characteristics}

The calibration experiment was designed in the following way: (a) Blank solution and seven standard solutions with a non-zero concentration were used in the concentration range $0-1.40 \mathrm{ppb} \mathrm{Pb}$ (II) $(I=8)$. (b) Four replicate measurements $(J=4)$ of the signal (DPASV current) were performed four each solution (including blank) so that altogether $n=I J=8 \times 4=32$ measurements were performed. (c) Two additional signal measurements were made for the calculations of the LOD and LOQ values by "traditional approach" so that altogether six blank measurements were made and $n_{b}=6$ was used in eqs. (3) and (4). (d) The obtained values of signal and concentration were processed by linear regression and the corresponding quantities were calculated using the equations shown above. Since the regression calculation in "traditional approach" is usually made without the blank measurements (which create here a distinct data set), the blank signals were not included so that $n=I J=7 \times 4=28$ in this case.

The most important results and values characterizing the measurement performance, mainly LOD, LOQ, CV and MDV, obtained for determination of lead in surface water, are summarised in Table 2 together with the needed auxiliary quantities. 
Several important inferences are available when exploring the results shown in Table 2 (where in its entries more decimal figures are left than necessary only due to a better comparison): (a) Exactly equal LOD and CV values are not surprising since by a thorough inspection of eqs. (5) and (9) it can be found that these equations are identical. (b) In the given case, the LOD calculated by traditional approach is not very different from the correct LOD (and CV) value but it may be by chance more different, higher or lower, since it depends on the differences between $s_{b}$ and $s_{y x}$ as well as 3 and $t(v, 0.99)$. (c) The found minimal detectable concentration is a bit smaller than the doubled LOD value but the difference is small $(0.73 \%)$; it is explained by the fact that the non-centrality parameters $\delta$ of the non-central $t$-distribution are not too different from the doubled critical $t$ - values assuming equal $\alpha=\beta$ and the same (not too small) number of degrees of freedom.

Table 2. Determination of lead by DPASV - comparison of the LOD and LOQ values with the critical value, $\mathrm{CV}$, and the minimum detectable value, $\mathrm{MDV}^{*}$

\begin{tabular}{|c|c|c|c|c|c|}
\hline \multicolumn{2}{|c|}{$\begin{array}{c}\text { Traditional } \\
\text { approach }\end{array}$} & \multicolumn{2}{|c|}{$\begin{array}{c}\text { ULA } \\
\text { (IUPAC recommended) }\end{array}$} & \multicolumn{2}{|c|}{ ISO } \\
\hline$n_{b}$ & 6 & $n$ & 32 & $n$ & 32 \\
\hline $\bar{y}_{b}$ & 19.2917 & $v$ & 30 & $v$ & 30 \\
\hline$s_{b}$ & 0.47726 & $s_{y x}$ & 0.58427 & $\hat{\sigma}$ & 0.58427 \\
\hline $3 s_{b}$ & 1.4318 & & & $I$ & 8 \\
\hline $10 s_{b}$ & 4.7726 & & & $J$ & 4 \\
\hline$q_{0}$ & 19.5186 & $q_{0}$ & 19.4067 & $q_{0}$ & 19.4067 \\
\hline \multirow[t]{7}{*}{$q_{1}$} & 7.2437 & $q_{1}$ & 7.3557 & $q_{1}$ & 7.3557 \\
\hline & & $t(v, 0.99)$ & 2.457 & $t(v, 0.99)$ & 2.457 \\
\hline & & & & $t(v, 0.95)$ & 1.697 \\
\hline & & $k_{D}$ & 3.741 & $\delta(v, 0.01,0.01)$ & 4.879 \\
\hline & & $k_{Q}$ & 11.223 & $\delta(v, 0.05,0.05)$ & 3.367 \\
\hline & & $\bar{x}$ & 0.7000 & $\bar{x}$ & 0.7000 \\
\hline & & $s_{x x}$ & 6.7200 & $s_{x x}$ & 6.7200 \\
\hline LOD, ppb & 0.1977 & LOD, ppb & 0.2051 & $\mathrm{CV}\left(x_{C}\right), \mathrm{ppb}$ & 0.2051 \\
\hline LOI, ppb & 0.3953 & $2 \mathrm{LOD}, \mathrm{ppb}$ & 0.4102 & $\operatorname{MDV}\left(x_{D}\right), \mathrm{ppb}$ & 0.4072 \\
\hline LOQ, ppb & 0.6589 & LOQ, ppb & 0.6153 & & \\
\hline
\end{tabular}

*) The measured quantity with the corresponding unit of the blank signal mean $\bar{y}_{b}$ and the blank standard deviation $s_{b}$ is the maximum DPASV peak current in nA. The ratio of the signal unit to the concentration unit (ppb) makes the slope unit. The LOI and LOQ in traditional approach are defined as $6 s_{b} / q_{1}$ and $10 s_{b}$ $/ q_{1}$, resp. The residual standard deviation is defined as:

$$
\hat{\sigma}=\sqrt{\frac{1}{I \cdot J-2} \sum_{i=1}^{I} \sum_{j=1}^{J}\left(y_{i j}-\hat{q}_{0}-\hat{q}_{1} x_{i}\right)^{2}} .
$$

The most needed values $\delta$ can be found in Table 3 otherwise $2 t(v, 1-\alpha)$ may be approximately used. (d) If $\alpha=\beta=0.05$ were used in the ISO approach then the 
resulting MDV would be smaller by $31 \%$ however, it was justified in the IUPAC recommendation (MOCAK et al., 1997) that a better choice for chemical measurements should be $\alpha=0.01$ otherwise the LOD and further limits used in chemical trace analysis would be too low, i.e. too optimistic.

The best design of the calibration experiment is equidistant with at least one standard, except the blank, located in the region around the LOQ. The equidistant design allows using of very simple eqs. $(9 \mathrm{a}, \mathrm{b})$ with the $k_{D}$ factor found in the last column of Table 1 . The usage of the calibration points at the concentration levels much higher than the limits of detection and quantification is sometimes made in practice but is not permissible (MASSART et al., 1988) because of errors caused by a distant extrapolation. Therefore very often a special calibration experiment is necessary for determining the LOD and LOQ, which is different from that used in a routine laboratory work.

\subsection{Recommendation what limits to use}

As mentioned in the first two parts of this work, there are nowadays known and defined several performance characteristics of a measurement process in chemical trace analysis due to the existence of IUPAC as well as ISO standards, recommendations and guidelines. Therefore it is reasonable to select those of them which are most important with regard to current trends of their utilization. Consequently, we would like to propose three main performance characteristics for a general use: (1) the limit of detection, LOD, as an equivalent of CV but much more widespread in chemical literature, (2) the minimum detectable value, MDV, the ISO quantity widely widespread in science and technology, (3) the limit of quantification, LOQ, as an unique chemical characteristic relevant to the smallest measure at which quantitative analysis is possible.

\section{Conclusions}

Newer way of calculation of the limit of detection, LOD, and limit of quantification, LOQ, recommended by IUPAC (MOCAK et al., 1997), overcomes the problems caused by statistical incorrectness of by now still used traditional way of calculation and can be applied easily as shown in this paper. Correct LOD and LOQ values can be obtained by equations (5) and (6) or, which is the simplest way, using eqs. (8) and $(9 a, b)$ under condition that an equidistant calibration design is realized. In such a case the proportionality factor $k_{D}$ can be found from Table 1 and the $k_{Q}$ factor is computed as its triple. The ISO defined critical value, $\mathrm{CV}$, is identical to the LOD defined by eq. (5). The minimum detectable value, MDV, is calculated by means of eq. (11) using the relevant non-centrality parameter $\delta$ of the non-central $t$-distribution, accessible in Table 3 for common cases. Otherwise the non-centrality parameter $\delta$ can be approximated by the doubled critical $t$ - values assuming equality $\alpha=\beta$ and the same number of degrees of freedom $v$. Under these conditions 2 LOD represents a good MDV approximation. The calculation possibilities are demonstrated in Table 2. 
Table 3. Values of the non-centrality parameter $\delta(v, \alpha, \beta)$ of the non-central $t$-distribution for $v$ degrees of freedom and significance levels $\alpha$ and $\beta$ (here $\alpha=\beta$ ).

\begin{tabular}{|c|c|c|c|c|c|}
\hline$v$ & $\delta(v, 0.05,0.05)$ & $\delta(v, 0.01,0.01)$ & $v$ & $\delta(v, 0.05,0.05)$ & $\delta(v, 0.01,0.01)$ \\
\hline 2 & 5.516 & 15.217 & 39 & 3.349 & 4.824 \\
\hline 3 & 4.456 & 9.338 & 40 & 3.347 & 4.819 \\
\hline 4 & 4.067 & 7.520 & 41 & 3.346 & 4.815 \\
\hline 5 & 3.870 & 6.683 & 42 & 3.344 & 4.811 \\
\hline 6 & 3.752 & 6.213 & 43 & 3.343 & 4.807 \\
\hline 7 & 3.673 & 5.915 & 44 & 3.342 & 4.803 \\
\hline 8 & 3.617 & 5.710 & 45 & 3.341 & 4.800 \\
\hline 9 & 3.575 & 5.562 & 46 & 3.339 & 4.797 \\
\hline 10 & 3.543 & 5.449 & 47 & 3.338 & 4.793 \\
\hline 11 & 3.517 & 5.361 & 48 & 2.337 & 4.790 \\
\hline 12 & 3.496 & 5.290 & 49 & 3.336 & 4.787 \\
\hline 13 & 3.479 & 5.232 & 50 & 3.335 & 4.785 \\
\hline 14 & 3.464 & 5.184 & 51 & 3.334 & 4.782 \\
\hline 15 & 3.451 & 5.143 & 52 & 3.334 & 4.779 \\
\hline 16 & 3.440 & 5.108 & 53 & 3.333 & 4.777 \\
\hline 17 & 3.431 & 5.077 & 54 & 3.332 & 4.774 \\
\hline 18 & 3.423 & 5.051 & 55 & 3.331 & 4.772 \\
\hline 19 & 3.415 & 5.027 & 56 & 3.330 & 4.770 \\
\hline 20 & 3.408 & 5.006 & 57 & 3.330 & 4.768 \\
\hline 21 & 3.402 & 4.987 & 58 & 3.329 & 4.766 \\
\hline 22 & 3.397 & 4.971 & 59 & 3.328 & 4.764 \\
\hline 23 & 3.392 & 4.955 & 60 & 3.328 & 4.762 \\
\hline 24 & 3.388 & 4.942 & 70 & 3.322 & 4.746 \\
\hline 25 & 3.383 & 4.929 & 80 & 3.318 & 4.734 \\
\hline 26 & 3.380 & 4.917 & 90 & 3.315 & 4.724 \\
\hline 27 & 3.376 & 4.907 & 100 & 3.312 & 4.717 \\
\hline 28 & 3.373 & 4.897 & 120 & 3.309 & 4.706 \\
\hline 29 & 3.370 & 4.888 & 150 & 3.305 & 4.695 \\
\hline 30 & 3.367 & 4.879 & 200 & 3.301 & 4.685 \\
\hline 31 & 3.365 & 4.871 & 300 & 3.297 & 4.674 \\
\hline 32 & 3.362 & 4.864 & 400 & 3.295 & 4.669 \\
\hline 33 & 3.360 & 4.857 & 500 & 3.294 & 4.665 \\
\hline 34 & 3.358 & 4.851 & 600 & 3.293 & 4.663 \\
\hline 35 & 3.356 & 4.845 & 700 & 3.293 & 4.662 \\
\hline 36 & 3.354 & 4.839 & 800 & 3.293 & 4.661 \\
\hline 37 & 3.352 & 4.834 & 1000 & 3.292 & 4.659 \\
\hline 38 & 3.350 & 4.829 & $\infty$ & 3.290 & 4.653 \\
\hline
\end{tabular}

Acknowledgement: Financial support of this work by the grants VEGA 1/1005/09 and VVCE-0004-07 is highly acknowledged.

\section{REFERENCES}

ACS Committee of Environmental Improvement: Guidelines for Data Acquisition and Data Quality Evaluation in Environmental Chemistry. Anal. Chem., 52, 1980, 2242-2249. 
CURRIE, L.A., HORWITZ, W.: IUPAC recommendations for defining and measuring detection and Quantification limits. Analysis, 22, 1994, m24-m26.

CURRIE, L.A., SVEHLA, G.: Nomenclature for the presentation of results of chemical analysis. Pure Appl. Chem., 66, 1994, 595-608.

CURRIE L. A.: Nomenclature in evaluation of analytical methods including detection and quantification capabilities. IUPAC Recommendations 1995. Pure Appl. Chem., 67, 1995, 1699-1723.

GREEN, J.R., MARGERISON, D.: (1978). Statistical Treatment of Experimental Data. Amsterdam. Elsevier. Chapter 14.3.

ISO 11843-2:2000: Capability of detection - Part 2: Methodology in the linear calibration case. International Organization for Standardization (01-May-2000). 24 pp. Corrigendum: ISO 11843-2/Cor1:2007.

IUPAC, ANALYTICAL CHEMISTRY DIVISION: Nomenclature, symbol, units and their usage in spectrochemical analysis. II. Data interpretation. Spectrochim. Acta, 33 B, 1978, 241-246.

IUPAC: Comission V5 web site (1999): http://users.unimi.it/ape/V5.

JANIGA, I., GARAJ, I., CISKO, P.: Estimation of Minimum Detectable Value by Using Linear Calibration with Constant Standard Deviation. Forum Metricum Slov., 8, 2004, 68-73.

JANIGA, I., MOCAK, J., CISKO, P., GARAJ, I., SZARKOVÁ, D.: Detection of the mercury content in food and medicines by means of linear calibration funktion. Forum Statisticum Slov., 2, 2006, 67-72.

MASSART D.L., VANDEGINSTE, B.G.M., DEMING, S.N., MICOTTE, Y., KAUFMAN, L.: Chemometrics: a Textbook. Elsevier, Amsterdam, 1988, p. 86-91; p. 113-114.

MOCAK, J., VARGA, S., POLAK, P., GERGELY, S., IZAK, J.: Calibration - Some newer chemometrical aspects in instrumental analysis. Wissenschaftliche Zeitsch., 32, 1990, 43-49.

MOCAK, J., BOND, A. M., MITCHELL, S., SCHOLLARY, G.: A Statistical Overview of Standard (IUPAC and ACS) and New Procedures for Determining the Limits of Detection and Quantification: Application to Voltammetric and Stripping Techniques. Pure Appl. Chem., 69, 1997, 297-328.

MOCAK, J., BOBROWSKI, A. Determination of cadmium and lead in water - new recommended way of evaluating the limits of detection and quantification. Water Sci. Technol., 1, 2001, 19-26.

STN ISO 11843-2: Detekčná schopnost'. Čast' 2: Metodika lineárnej kalibrácie (in Slovak). STN (Slovak Technical Standard), Bratislava, 2002. 